\title{
Community perceptions of bloody diarrhoea in an urban slum in South Asia: implications for introduction of a Shigella vaccine
}

\author{
W. ARVELO ${ }^{1 *}$, L. S. BLUM ${ }^{2}$, N. NAHAR ${ }^{2}$, L. VON SEIDLEIN ${ }^{3}$, L. NAHAR ${ }^{2}$, \\ R. P. PACK ${ }^{4}$, A. W. BROOKS ${ }^{2}$, A. PACH ${ }^{3}$, R. F. BREIMAN ${ }^{1}$, S. P. LUBY \\ AND P. K. RAM ${ }^{1,5}$ \\ ${ }^{1}$ Centers for Disease Control and Prevention, Atlanta, GA, USA \\ ${ }^{2}$ ICDDR,B: International Centre for Diarrhoeal Disease Research, Dhaka, Bangladesh \\ ${ }^{3}$ International Vaccine Institute, Seoul, Republic of South Korea \\ ${ }^{4}$ East Tennessee State University, College of Public Health, TN, USA \\ ${ }^{5}$ University at Buffalo, Buffalo, USA
}

(Accepted 20 May 2010; first published online 14 June 2010)

\section{SUMMARY}

Understanding local perceptions of disease causation could help public health officials improve strategies to prevent bloody diarrhoea. A cross-sectional survey was conducted in Dhaka, Bangladesh to elicit community beliefs about the causes of and prevention strategies for bloody diarrhoea. Between March and June 2003, we interviewed 541 randomly selected respondents. Overall, 507 (93\%) respondents perceived that a vaccine could prevent bloody diarrhoea. If a vaccine provided lifetime protection, $445(83 \%)$ respondents stated that they would opt to get the vaccine and would pay a median of $\$ 0 \cdot 05$ (range U.S.\$0.01-0.15) for it, equivalent to $<1 \%$ of their median weekly income. There was almost universal perception that an effective vaccine to prevent bloody diarrhoea was highly beneficial and acceptable. While respondents valued a vaccine for prevention of bloody diarrhoea, they were only willing to pay minimally for it. Therefore, achieving a high rate of Shigella vaccine coverage may require subsidy of vaccine purchase.

Key words: Bloody diarrhoea, knowledge, perceptions, South Asia, vaccine.

\section{INTRODUCTION}

Bloody diarrhoea is endemic worldwide and is estimated to cause 165 million cases and over 1 million deaths each year; children comprise $69 \%$ of cases and $61 \%$ of deaths [1]. About $80-90 \%$ of all bloody diarrhoea is caused by Shigella spp. [2]. Shigella flexneri is the most frequently isolated species in developing countries [3]. However, only Shigella dysenteriae type

\footnotetext{
*Author for correspondence: W. Arvelo, M.D., M.Sc., Centers for Disease Control and Prevention, Unit 3190, Box 150, DPO, AA, 34024, Atlanta, GA, USA.

(Email:warvelo@gt.cdc.gov)
}

1 has been associated with large and deadly epidemics $[4,5]$. The only reservoirs for Shigella spp. are humans and primates [6]. Transmission occurs through the faecal-oral route, and most infections are transmitted from person-to-person through contact with contaminated objects [7]; houseflies may serve as vectors for faecal-oral transmission [8]. Because of the exceedingly low pathogen dose required for symptomatic infection, person-to-person transmission of Shigella is common. Persons living in areas with poor hand hygiene, inadequate sanitation facilities, and overcrowding are at increased risk for acquiring infection [9-11]. 
The clinical manifestations of Shigella-related diarrhoea vary from mild diarrhoeal illness to severe diarrhoea, including dysentery, that may result in complications such as malnutrition, toxic megacolon, intestinal perforation, and haemolytic-uraemic syndrome [12-14]. Management of the disease includes early rehydration, adequate nutrition, laboratory confirmation of the causative pathogen, and antimicrobial therapy $[3,15]$. Treatment of bloody diarrhoea has been complicated by increasing antimicrobial resistance in Shigella, leaving few costeffective and practical pharmaceutical options for curative care [16-18].

A variety of parenteral, oral, and nasally administered shigellosis vaccine formulations are currently under development for prevention of bloody diarrhoea [19-21]. Clinical and field trials have found several candidates to be potentially immunogenic and promising [22]. Results from formative research carried out in several Asian countries suggest that belief systems associated with bloody diarrhoea along with poor existing water and sanitation conditions may create a high demand for a shigellosis vaccine [23-25].

Understanding local beliefs about shigellosis and perceptions of vaccines is relevant for determining vaccine demand, developing culturally appropriate interventions to increase vaccine demand, and reducing potential barriers to vaccine delivery and acceptability [26]. We examined beliefs regarding bloody diarrhoea and the acceptability of a hypothetical vaccine to prevent bloody diarrhoea in residents of a densely populated urban slum of Dhaka, Bangladesh.

\section{METHODS}

\section{Site}

Kamalapur, spanning just over $4.0 \mathrm{~km}^{2}$ in southeastern Dhaka, has a population of $>200000$ persons. This densely populated community is typical of many rapidly growing urban slums in Dhaka. In addition to extremely high population density, the pressure for transmission of enteric pathogens is heightened by highly contaminated municipal water supplies, ubiquitous open untreated sewage runoff, and poor hand hygiene practices [27, 28].

\section{Procedures and study population}

We conducted a cross-sectional household survey between March and July, 2003. Households were selected using stratified cluster sampling with the selection of clusters proportional to population size as reported in available government census data. We determined that a sample size of 550 households would provide enough statistical power for this study. Of these 550 households, nine were excluded from the analysis because of missing data. A total of 541 households were identified and included in this study.

Once a household was selected, we randomly selected one person aged $\geqslant 15$ years living in the household for interview. Data were collected using a standardized close-ended questionnaire, which was developed based on the results of a multi-country qualitative study carried out in six South and East Asian countries. The qualitative study examined perceptions on severity, vulnerability, and causes of bloody diarrhoea; prevention and health-seeking practices for the disease, and acceptability of a shigellosis vaccine [24]. The study instrument was modified according to the local context and translated into Bengali. Pilot tests of the instrument were conducted in the study site for response category validity and accuracy.

Data collection was carried out by four interviewers who received extensive training in sampling procedures, interviewing, and taking consent before the study. We analysed data using Statistical Analysis System version 9.1 (SAS Institute, USA) and generated frequencies for categorical variables, and measures of central tendency for continuous variables. We conducted bivariate analyses to examine the association between independent and dependent variables; outcomes of interest included the perception regarding bloody diarrhoea and vaccine acceptability.

\section{Ethics}

Informed written consent was obtained from all study participants. The study protocol was approved by the Ethics Review Committee of the International Centre for Diarrhoeal Disease Research, Bangladesh and the Select Committee for Research on Human Subjects of the World Health Organization.

\section{RESULTS}

Of 541 respondents interviewed, the median age was 35 years (range $15-82$ years) and $276(51 \%)$ were female. The median household size was five persons (range 4-6 persons) and the median monthly income reported per household was U.S.\$105 (range 
Table 1. Perceived level of vulnerability to becoming ill with bloody diarrhoea by age groups, household community survey, Dhaka, Bangladesh, 2003

\begin{tabular}{llll}
\hline \hline & \multicolumn{3}{l}{ Level of vulnerability $(n=541)$} \\
\cline { 2 - 4 } Age group & High & Neutral & None \\
$n(\%)$ & $n(\%)$ & $n(\%)$ \\
\hline Infant & $207(38)$ & $151(28)$ & $183(34)$ \\
Pre-school children & $233(43)$ & $188(35)$ & $120(22)$ \\
Older children & $177(33)$ & $238(44)$ & $126(23)$ \\
Adults & $113(20)$ & $236(44)$ & $192(36)$ \\
Elderly & $271(50)$ & $183(34)$ & $87(16)$ \\
\hline \hline
\end{tabular}

U.S.\$60-175). Of male participants, 243 (92\%) reported being employed; $50(19 \%)$ had a small business (e.g. food seller, small shopkeeper, etc.), 26 (11\%) were rickshaw pullers, $24(10 \%)$ ran a large business (e.g. shopkeeper, hotel/restaurant, landlord, etc.), and $18(7 \%)$ were office attendants, sweepers, gardeners, or cooks. Of women participants, $211(77 \%)$ described themselves as full-time housewives, $17(6 \%)$ worked as maids, and eight (3\%) worked in embroidery. Electricity was available in $531(98 \%)$ households, and $127(24 \%)$ owned a television.

Taps from tube wells were the main water source for $325(60 \%)$ households, while $206(38 \%)$ reported receiving water from a municipal tap; 304 (56\%) respondents felt that water treatment in the community was adequate. Ring slab, open, or sanitary latrines were available in 536 (99\%) households; 316 (58\%) respondents felt that sanitation facilities were adequate, and $273(51 \%)$ reported that sewage disposal in the community was adequate. Almost all $(99 \%)$ respondents reported washing their hands after defecation. Only 191 (35\%) reported washing their hands after cleaning a child who had defecated and $157(29 \%)$ respondents reported washing their hands before handling food. Twenty-two (4\%) respondents reported using soap when washing their hands after using the toilet or before preparing or eating foods.

Seventy-eight $(15 \%)$ respondents thought bloody diarrhoea was a common problem in the community. The 'elderly' were most frequently reported to be 'very vulnerable to bloody diarrhoea' (Table 1). Respondents thought it was 'very likely' $(9 \%)$ or 'somewhat likely' $(65 \%)$ for someone in the household to have had bloody diarrhoea. A third of the participants reported that someone in the household had at some point been sick with bloody diarrhoea, and $11(2 \%)$ were aware of a death in the community
Table 2. Perceived level of socioeconomic impact to the household of bloody diarrhoea in household members, by age group and sex, Dhaka, Bangladesh, 2003

\begin{tabular}{lllr}
\hline \hline & \multicolumn{2}{l}{$\begin{array}{l}\text { Level of socioeconomic impact } \\
\text { to the household }(n=541)\end{array}$} \\
\cline { 2 - 4 } $\begin{array}{l}\text { Household } \\
\text { member }\end{array}$ & $\begin{array}{l}\text { High } \\
n(\%)\end{array}$ & $\begin{array}{l}\text { Neutral } \\
n(\%)\end{array}$ & $\begin{array}{r}\text { None } \\
n(\%)\end{array}$ \\
\hline $\begin{array}{l}\text { Female child } \\
\text { Male child }\end{array}$ & $300(55)$ & $93(17)$ & $9(2)$ \\
Female adult & $326(60)$ & $88(16)$ & $9(2)$ \\
Male adult & $388(72)$ & $173(32)$ & $14(3)$ \\
\hline \hline
\end{tabular}

associated with bloody diarrhoea. Approximately half of the respondents perceived bloody diarrhoea to be a severe disease; 280 (52\%) thought bloody diarrhoea was more severe than other diarrhoeal syndromes, and $248(46 \%)$ perceived bloody diarrhoea as a more severe problem than other health problems in the community. Three hundred and eighty-eight ( $72 \%)$ respondents reported that the socioeconomic consequences for the household were 'high' when bloody diarrhoea affects adult men (Table 2). This level of socioeconomic consequence was reported less frequently when bloody diarrhoea affects adult women $(64 \%)$, male children $(60 \%)$, and female children $(55 \%)$ (Table 2). When asked who should receive a vaccine against bloody diarrhoea, $531(98 \%)$ respondents reported that a child aged $<5$ years should receive the vaccine. Moreover, 473 (87\%) individuals reported that an adult should receive the vaccine and $459(85 \%)$ respondents thought the elderly should receive it.

Respondents believed that the risk of bloody diarrhoea is increased by eating foods containing chilies $(93 \%)$, the presence of standing sewage near the household $(78 \%)$, drinking dirty or unboiled water $(73 \%)$, and not washing hands after defecating $(66 \%)$ (Table 3). Strategies perceived to prevent bloody diarrhoea included consuming 'cold foods' $(58 \%)$, improving the water supply $(48 \%)$, disposing of faeces in a sanitary fashion $(47 \%)$, and avoiding foods containing chilies $(41 \%)$. When compared to respondents who had no education, respondents who reported receiving any level of education were significantly more likely to associate drinking dirty or unboiled water $(P<0.05)$ and not washing hands after defecating $(P=0.05)$ as causes of bloody diarrhoea (Table 4). 
Table 3. Perceived causes and corollary perceived preventive strategies for bloody diarrhoea, household community survey, Dhaka, Bangladesh, $2003(n=541)$

\begin{tabular}{llll}
\hline \hline Perceived cause of bloody diarrhoea & $n(\%)$ & Perceived prevention strategy & $n(\%)$ \\
\hline Eating foods with chili & $502(93)$ & Eating cold foods & $351(58)$ \\
Standing sewage near household & $424(78)$ & Improved disposal of faeces & $253(47)$ \\
Drinking dirty, unboiled water & $385(73)$ & Increase access to safer water & $259(48)$ \\
Not washing hands after defecating & $357(66 \%)$ & & \\
\hline \hline
\end{tabular}

Table 4. Association between education level of the respondent and perceived causes of bloody diarrhoea, Dhaka, Bangladesh, 2003

\begin{tabular}{llll}
\hline \hline & \multicolumn{2}{l}{ Level of education } & \\
\cline { 2 - 4 } Perceived cause of bloody diarrhoea & Any, $n(\%)$ & None, $n(\%)$ & $P$ value \\
\hline Eating foods with chili & $309(96)$ & $192(95)$ & $0 \cdot 7$ \\
Standing sewage near the house & $267(82)$ & $252(80)$ & $0 \cdot 5$ \\
Drinking dirty or unboiled water & $265(83)$ & $129(70)$ & $<0 \cdot 05$ \\
Not washing hands after defecating & $288(86)$ & $160(80)$ & $0 \cdot 05$ \\
\hline \hline
\end{tabular}

Only $134(25 \%)$ households believed there was medical treatment for bloody diarrhoea. Overall, 507 $(93 \%)$ respondents perceived that a vaccine could prevent bloody diarrhoea, and $533(99 \%)$ reported willingness to receive a vaccine if one were available. When asked what additional information they would need to accept a vaccine, $379(70 \%)$ respondents wanted to receive information about potential adverse effects of the vaccine, and in these almost all respondents wanted to know about the potential for the vaccine to cause infertility. The vast majority of respondents also wanted to know about the specific delivery method of the vaccine (oral vs. injection), and virtually all respondents also desired information on the time between doses $(99 \%)$ and number of doses $(98 \%)$. Only $149(28 \%)$ respondents stated the need for information on vaccine effectiveness before deciding to get the vaccine. If a vaccine provided lifetime protection, $445(83 \%)$ households reported they would pay a median of U.S.\$0.05 (range \$0.01-0.15), equivalent to $<1 \%$ of their median weekly income, to get the vaccine for themselves or a family member.

\section{DISCUSSION}

This survey, carried out in an urban slum in South Asia, found that bloody diarrhoea was perceived as having negative clinical and socioeconomic impact on households. In our study population, few persons believed that medical treatment for bloody diarrhoea was available. Although many respondents did not identify effective preventive measures against bloody diarrhoea, such as having access to an improved water supply or improving hand hygiene, an effective vaccine to prevent bloody diarrhoea was perceived as beneficial and highly acceptable to most respondents. Despite these conceptions the study population was willing to pay only a small amount for the vaccine. Respondents would pay for a vaccine to prevent bloody diarrhoea if it were priced low enough and if the vaccine provided lifetime protection. The vaccine was considered acceptable and appropriate for all age groups particularly children aged $<5$ years.

Several factors may explain the high acceptability of a vaccine to prevent shigellosis in this community. First, perceived severity of illness has been shown to increase acceptance of biomedical treatments such as vaccines [29, 30]. Previous research in this community has suggested that the cultural significance of blood loss in the dysenteric stools, and the uncertainty surrounding treatment of bloody diarrhoea, may have induced a perception of extreme seriousness of the disease [23], and thus the high degree of willingness to accept a vaccine to prevent the disease. Second, perceptions of the potential socioeconomic consequences to the household if a member is afflicted by a disease can play a role in persons accepting a vaccine for the disease [24, 31]. The economic impact for the household was more frequently perceived to be very serious for bloody diarrhoea in an adult, compared to a child. These perceptions about the relative economic impact on the household for an adult with bloody diarrhoea 
are consistent with other studies, which have described immense costs in terms of lost productivity and income generation as a result of diseases such as tuberculosis, HIV, malaria, and dengue [30-32]. The perception of higher vulnerability in the elderly and the belief that the socioeconomic consequences to the households are highest when an adult male is affected with the disease, may represent difficulties in implementing a vaccine programme for children, who are at higher risk for enteric diseases. Satisfaction with previous immunization services have also been shown to increase acceptability to new vaccines [29]. Efforts in Bangladesh to create demand and provide services for universal immunization coverage have been highly successful, and together with the perception of severity regarding bloody diarrhoea and the belief that a vaccine could prevent the disease may have accounted for the high vaccine acceptability in this study [33, 34]. Many of the respondents were not aware of medical treatments for bloody diarrhoea, and this may have also led to increased acceptability for a vaccine.

Despite the high acceptability for the vaccine and perceptions regarding the severity of the disease, respondents were willing to pay only a small amount of their income to obtain the vaccine and protect household members against bloody diarrhoea, and they would get the vaccine only if the vaccine would provide lifetime protection. A recent study on varicella vaccination in China showed a high parental willingness to have their children immunized against the disease if the vaccines were provided free of charge or heavily subsidized [35], but other studies looking at private sector provision for vaccines such as typhoid have shown much higher willingness to pay than that found in this study population [36]. Currently Bangladesh's Expanded Programme of Immunization, which began in 1979, is successfully providing almost universal access to immunization services for several vaccine-preventable diseases in children [37]. Our study suggests that successful introduction of a Shigella vaccine in this or similar populations will require major subsidies, similar to those provided by the Expanded Programme of Immunization which fully subsidizes vaccines, in order to achieve high Shigella vaccine coverage [38].

Willingness to get the vaccine was conditional on several factors other than socioeconomic status. Parental perceptions of the risks of vaccines and preferences of parents and healthcare workers for the vaccine delivery type, have all been shown to be part of the framework within which vaccines are accepted by a community, particularly for childhood vaccination [39]. Respondents also stated that the vaccine delivery type (e.g. injection, oral, nasal) and the number of required doses were important considerations in the decision to accept to get the vaccine for themselves or their family members. These findings suggest that additional formative research on the specific types of vaccine delivery and dosing schedule acceptable to the community should be investigated before initiating the vaccine development process.

We also found that there were several misconceptions about the causal pathways for bloody diarrhoea. Over half of respondents believed that consuming 'cold foods' was an appropriate prevention strategy for bloody diarrhoea. Previous research has shown that 'cold foods' are believed to counteract a 'hot illness' such as bloody diarrhoea, suggesting that in this population humeral theories play a major role in the local perceptions of disease causation [23]. Persons with lower levels of education, who are presumably of lower socioeconomic status and at higher risk for diarrhoeal diseases including bloody diarrhoea, were more likely to have misconceptions regarding disease aetiology and preventative measures for bloody diarrhoea. Despite these misconceptions there was high acceptability for a vaccine, and this is in keeping with other studies in Africa and Asia that have found no associations between knowledge of a disease and vaccine acceptability [24, 40, 41].

Many potential difficulties exist for successfully implementing, maintaining, and scaling-up a vaccination programme. Adding new vaccines to ongoing childhood vaccine programmes may overburden the already limited cold chain and storage capacities for vaccines in developing countries. Moreover, distribution and implementation of new vaccines require substantial financial support that may be limited in many developing countries. Availability of safe water, reliable sewage disposal facilities, and good hand washing practices are essential strategies to reduce diarrhoea morbidity in developing countries [27, 42, 43]. Strategies to improve hygiene infrastructure and modify behaviours are presently being implemented in Bangladesh, but they require much time and investment $[28,44,45]$. Given the prolonged time required to improve national public infrastructures for water, hygiene, and sanitation, and the inherent difficulties in implementing prevention programmes that target behaviour modifications such as hand washing and treatment of household drinking water, vaccines 
represent an attractive complementary intervention to prevent childhood morbidity and death from enteric diseases.

Our findings are subject to two principal limitations. First, although our study sample was drawn from a community typical in Dhaka with socioeconomic and environmental conditions that predispose residents to a high risk of diarrhoeal diseases, this community may not represent the general population of Bangladesh; however, those living in Kamalapur represent a fairly typical urban population that would benefit from an effective vaccine, and the participants were selected in a manner that aimed to reduce selection bias. Second, questions regarding vaccine acceptability may have been subject to courtesy bias, since the data collectors were employees of ICDDR,B, a well-recognized biomedical research establishment in Bangladesh and the community.

Despite these limitations, this study documents a high degree of acceptability for a Shigella vaccine. Nonetheless, while respondents recognized a significant value for a vaccine to prevent Shigella dysentery, respondents were willing to pay only a small amount for the vaccine. Effective prevention efforts to prevent bloody diarrhoea in a poor region in South East Asia via immunizations will probably need to be linked to heavily subsidized programmes targeted to populations at greatest risk.

\section{ACKNOWLEDGEMENTS}

We gratefully acknowledge the participation of the study population in Dhaka, as well as the contributions of Dr Bonita Stanton and Dr Eric Mintz. This study was supported by funds from the Bill and Melinda Gates Foundation through the Diseases of the Most Impoverished Program of the International Vaccine Institute, and by funds for the Institute from the governments of the Republic of Korea, Sweden, and Kuwait.

\section{DECLARATION OF INTEREST}

None.

\section{REFERENCES}

1. Kotloff KL, et al. Global burden of Shigella infections: implications for vaccine development and implementation of control strategies. Bulletin of the World Health Organization 1999; 77: 651-666.

2. Wittenberg DF. Emerging and re-emerging diseases epidemic enterohaemorrhagic infections 100 years after Shiga. South Africa Medical Journal 1999; 89: $750-752$.

3. von Seidlein L, et al. A multicentre study of Shigella diarrhoea in six Asian countries: disease burden, clinical manifestations, and microbiology. PLoS Medicine 2006; 3 : 353 .

4. CDC. Health status of displaced persons following Civil War-Burundi, December 1993-January 1994. Morbidity and Mortality Weekly Report 1994; 43: 701-703.

5. Rahaman MM, et al. An outbreak of dysentery caused by Shigella dysenteriae type 1 on a coral island in the Bay of Bengal. Journal of Infectious Diseases 1975; 132: $15-19$.

6. Niyogi SK. Shigellosis. Journal of Microbiology 2005; 43: 133-143.

7. DuPont HL, et al. Inoculum size in shigellosis and implications for expected mode of transmission. Journal of Infectious Diseases 1989; 159: 1126-1128.

8. Levine OS, Levine MM. Houseflies (Musca domestica) as mechanical vectors of shigellosis. Review of Infectious Diseases 1991; 13: 688-696.

9. Anon. Public health impact of Rwandan refugee crisis: what happened in Goma, Zaire, in July, 1994? Goma Epidemiology Group. Lancet 1995; 345: 339-344.

10. CDC. Outbreaks of multidrug-resistant Shigella sonnei gastroenteritis associated with day care centers Kansas, Kentucky, and Missouri 2005. Morbidity and Mortality Weekly Report 2006; 55: 1068-1071.

11. Mohle-Boetani JC, et al. Communitywide shigellosis: control of an outbreak and risk factors in child day-care centers. American Journal of Public Health 1995; 85: 812-816.

12. Bennish ML, Wojtyniak BJ. Mortality due to shigellosis: community and hospital data. Review of Infectious Diseases 1991; 13 (Suppl. 4): 245-251.

13. Koster F, et al. Hemolytic-uremic syndrome after shigellosis. Relation to endotoxemia and circulating immune complexes. New England Journal of Medicine 1978; 298: 927-933.

14. van den Broek JM, et al. Risk factors for mortality due to shigellosis: a case-control study among severelymalnourished children in Bangladesh. Journal of Health, Population and Nutrition 2005; 23: 259-265.

15. Chao HC, et al. Bacterial enteric infections in children: etiology, clinical manifestations and antimicrobial therapy. Expert Review of Anti-Infective Therapy 2006; 4: 629-638.

16. Finkelstein JA, et al. Antimicrobial use in defined populations of infants and young children. Archives of Pediatrics \& Adolescent Medicine 2000; 154: 395-400.

17. Jain R, Danziger LH. The macrolide antibiotics: a pharmacokinetic and pharmacodynamic overview. Current Pharmaceutical Design 2004; 10: 3045-3053.

18. Sivapalasingam S, et al. High prevalence of antimicrobial resistance among Shigella isolates in the 
United States tested by the National Antimicrobial Resistance Monitoring System from 1999 to 2002. Antimicrobial Agents and Chemotherapy 2006; 50: 49-54.

19. Coster TS, et al. Vaccination against shigellosis with attenuated Shigella flexneri 2a strain SC602. Infection and Immunity 1999; 67 : 3437-3443.

20. Jennison AV, Verma NK. Shigella flexneri infection: pathogenesis and vaccine development. FEMS Microbiology Reviews 2004; 28 : 43-58.

21. Kotloff KL, et al. Safety, immunogenicity, and transmissibility in humans of CVD 1203, a live oral Shigella flexneri 2 a vaccine candidate attenuated by deletions in aroA and virG. Infection and Immunity 1996; 64: 4542-4548.

22. Phalipon A, Mulard LA, Sansonetti PJ. Vaccination against shigellosis: is it the path that is difficult or is it the difficult that is the path? Microbes and Infection 2008; 10: 1057-1062.

23. Blum LS, Nahar N. Cultural and social context of dysentery: implications for the introduction of a new vaccine. Journal of Health, Population and Nutrition 2004; 22 : 159-169.

24. Pack R, et al. Willingness to be vaccinated against shigella and other forms of dysentery: a comparison of three regions in Asia. Vaccine 2006; 24: 485-494.

25. Youlong G, et al. Perceptions of Shigella and of Shigella vaccine among rural Chinese: compatibility with Western models of behavioral change. Southeast Asian Journal of Tropical Medicine and Public Health 2004; 35: $97-108$.

26. Jheeta M, Newell J. Childhood vaccination in Africa and Asia: the effects of parents' knowledge and attitudes. Bulletin of the World Health Organization 2008; 86: 419 .

27. Esrey SA, Habicht JP. Epidemiologic evidence for health benefits from improved water and sanitation in developing countries. Epidemiologic Reviews 1986; 8: 117-128.

28. Ram PK, et al. Risk factors for typhoid fever in a slum in Dhaka, Bangladesh. Epidemiology and Infection 2007; 135: 458-465.

29. Chen X, et al. Differences in perception of dysentery and enteric fever and willingness to receive vaccines among rural residents in China. Vaccine 2006; 24: 561-571.

30. Clark DV, et al. Economic impact of dengue fever/ dengue hemorrhagic fever in Thailand at the family and population levels. American Journal of Tropical Medicine and Hygiene 2005; 72: 786-791.

31. Muniyandi M, et al. Socio-economic dimensions of tuberculosis control: review of studies over two decades from Tuberculosis Research Center. Journal of Communicable Diseases 2006; 38: 204-215.
32. Russell S. The economic burden of illness for households in developing countries: a review of studies focusing on malaria, tuberculosis, and human immunodeficiency virus/acquired immunodeficiency syndrome. American Journal of Tropical Medicine and Hygiene 2004; $71:$ 147-155.

33. Anon. Expanded Programme on Immunization (EPI). The Social Science and Immunization Research Project. Weekly Epidemiological Record 1998; 73: 285-288.

34. Streefland P, Chowdhury AM, Ramos-Jimenez P. Patterns of vaccination acceptance. Social Science and Medicine 1999; 49: 1705-1716.

35. Liao SL, et al. Survey of the status of self-paid varicella vaccination among children one to six years of age in Taiwan. Journal of Microbiology, Immunology and Infection 2007; 40: 112-115.

36. Do GC, et al. Household demand for typhoid fever vaccines in Hue, Vietnam. Health Policy Plan 2006; 21 : 241-255.

37. Jamil K, et al. The immunization programme in Bangladesh: impressive gains in coverage, but gaps remain. Health Policy Plan 1999; 14: 49-58.

38. Levin A. Charging for family planning and $\mathrm{MCH}$ commodities and services: mid-term evaluation, $\mathrm{MCH}$ FP extension project (rural). International Centre for Diarrhoeal Disease Research, Bangladesh, ICDDR'B, 1997 (Working Paper No. 93.).

39. Forrest JM, Burgess MA, McIntyre PB. Factors influencing vaccination uptake. Workshop report. Current Australian research on the behavioural, social and demographic factors influencing immunisation, Royal Alexandra Hospital for Children, Sydney, March 1998. Communicable Disease Intelligence 2000; 24: 51-53.

40. Sauerborn R, et al. Willingness to pay for hypothetical malaria vaccines in rural Burkina Faso. Scandinavian Journal of Public Health 2005; 33: 146-150.

41. Kaljee LM, et al. Acceptability and accessibility of a Shigellosis vaccine in Nha Trang city of Viet Nam. Journal of Health, Population and Nutrition 2004; 22 : 150-158.

42. Checkley W, et al. Effect of water and sanitation on childhood health in a poor Peruvian peri-urban community. Lancet 2004; 363: 112-118.

43. van Poppel F, van der Heijden C. The effects of water supply on infant and childhood mortality: a review of historical evidence. Health Transition Review 1997; 7: 113-148.

44. Anon. Water and sanitation: the neglected health MDG. Lancet 2006; 368: 1212.

45. Clasen T, et al. Household water treatment using sodium dichloroisocyanurate (NaDCC) tablets: a randomized, controlled trial to assess microbiological effectiveness in Bangladesh. American Journal of Tropical Medicine and Hygiene 2007; 76: 187-192. 